\title{
Behavioral Inhibition: Temperament or Prodrome?
}

\author{
Koraly E. Pérez-Edgar • Amanda E. Guyer
}

Published online: 24 June 2014

(C) Springer International Publishing AG 2014

\begin{abstract}
Individual differences in temperament emerge in the first months of life. Some infants display a heightened sensitivity to novelty and uncertainty in the world around them, leading a subset to fearfully withdraw from the social environment. Extreme forms of this temperament, Behavioral inhibition (BI), are associated with increased risk for social anxiety disorder. Indeed, the link is so strong that some suggest that $\mathrm{BI}$ is not simply a risk factor for anxiety, but rather a milder form of the disorder. The current overview describes the literature linking $\mathrm{BI}$ and anxiety, highlighting the unique biobehavioral profiles evident in each construct. It then highlights specific evidence that may help distinguish the form and function of BI and anxiety. Finally, we briefly discuss unresolved issues that may help inform future work aimed at improving our understanding of individual development and shape therapeutic interventions directed at specific mechanisms of disorder.
\end{abstract}

Keywords Temperament $\cdot$ Social anxiety $\cdot$ Developmental psychopathology $\cdot$ Socioemotioanl development $\cdot$ Biological mechanisms

\section{Introduction}

Learning to navigate the social world is a core developmental task. To do so adaptively, children must create for themselves a stable understanding of who they are as individuals and their

K. E. Pérez-Edgar $(\bowtie)$

Department of Psychology, Child Study Center,

The Pennsylvania State University,

270 Moore Building, University Park, PA 16802, USA

e-mail: kxp24@psu.edu

A. E. Guyer

Department of Human Ecology, Center for Mind and Brain,

University of California, Davis, USA place within the specific social context that surrounds them. One need not be a trained psychologist to note the wide individual differences displayed by children, and later adults, as they come to find their place in the world. Indeed, we have centuries of writing and debate attempting to explain why some children eagerly embrace the ambiguities and uncertainties of their environments as opportunities for discovery and surprise, while other children retreat from the world, overwhelmed by an unpredictable landscape they see as marked by signs of threat and risk.

This article focuses on behavioral inhibition (BI), an early appearing temperamental trait that is marked by sensitivity to novelty and linked to the later emergence of psychopathology, particularly social anxiety $[1,2]$. Specifically, recent work suggests that by mid-adolescence behaviorally inhibited children have a fourfold increase in risk for social anxiety disorder [3], particularly when levels of BI are high and stable over time [4]. The association between temperament traits, particularly $\mathrm{BI}$, and psychopathology, in this case social anxiety, is considered a core conduit between early individual differences and the later emergence of disorder. Indeed, both The Handbook of Temperament and The Handbook of Developmental Psychopathology have chapters dedicated to the association between temperament and disorder $[5 \bullet, 6 \bullet]$. However, questions still remain regarding the definition and conceptualization of temperament vs. disorder and the mechanisms that may link the two constructs (if indeed they are separable constructs).

The present discussion defines and characterizes the current understanding of BI at the level of behavior and biology, and reviews the evidence linking BI to social anxiety. We then address the nature of the relation between BI and social anxiety, as several models have alternatively suggested that BI and social anxiety are associated but not causally linked constructs, that BI serves as a diathesis for social anxiety, and finally, that BI is a milder, prodromal phase before the onset of acute, impairing social anxiety (Fig. 1). In doing so, we will outline processes and mechanisms that seem to bind the two 
constructs, as well as potential areas of distinction. Finally, we note how a developmental perspective, incorporating multiple levels of analysis over time, may help clarify the conceptual and structural questions that remain.

\section{Behavioral Inhibition as a Form of Temperament}

The Greek physician Galen, building on the work of Hippocrates, argued that individuals could be characterized by one of

(A)

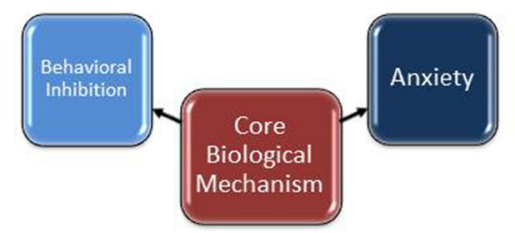

(B)

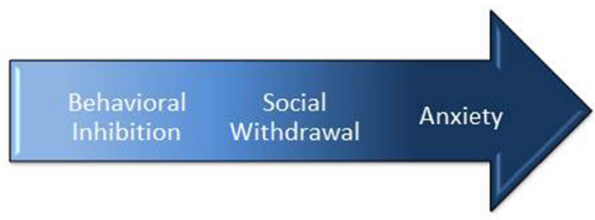

(C)
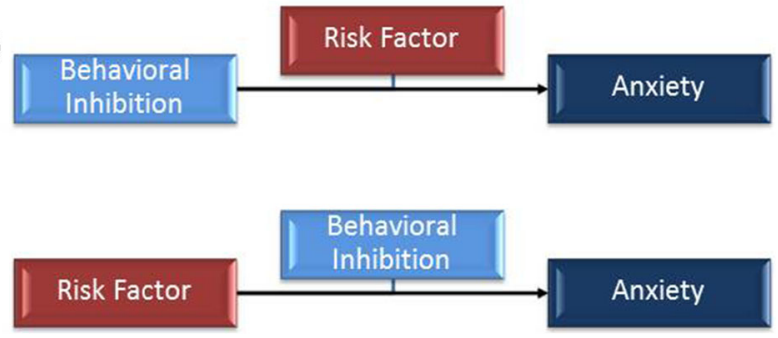

Fig. 1 Schematic representations of the proposed relation between Behavioral Inhibition (BI) and anxiety. There are strong empirical data based on longitudinal and cross-sectional studies linking BI to the emergence of anxiety, particularly social anxiety. In characterizing this relation, a number of models have been offered to describe the nature of this relation. (a) Individuals who show early BI are at increased risk for anxiety due to a shared core biological mechanism that generates both constructs. However, there is no direct causal relation between BI and anxiety. (b) Again, $\mathrm{BI}$ and anxiety are not causally related. Rather, BI and anxiety fall on a shared spectrum of socioemotional functioning. Children who "land" on the BI portion of the spectrum will remain inhibited, and healthy, while children in the anxiety portion will show signs of disorder. Alternatively, BI can be viewed as a "way station" in the developmental trajectory towards anxiety, such that a child who initially lands on the BI spectrum will over time show increases in social withdrawal and then progress toward anxiety. (c) The third set of models purports that BI plays a causal role in the emergence of anxiety, but stresses that they are separate constructs. In one form, BI acts as a diathesis that sets the foundation for anxiety, which in turn may be triggered by the shared presence of a risk factor, such as over-solicitous parenting styles. In a related model, BI acts as the moderator, shaping the manner in which underlying risk factors are manifest as psychopathology. For example, genetic risk for maladaptation may present as social anxiety when an individual also has high levels of BI. However, in the absence of BI, maladjustment may be evident in elevated levels of depression four dominant temperaments - sanguine, melancholic, choleric, and phlegmatic - that reflected stable patterns of behavior and emotion. Each temperament type, in turn, reflected a unique underlying balance of four bodily fluids - blood, yellow bile, black bile, and phlegm. When the fluids became imbalanced, the individual would manifest extreme distress and discomfort. Our modern conversation concerning temperament is remarkably similar in its outline, although we have come to substitute the concept of neurotransmitters for bodily fluids, and now give extreme distress and discomfort the label of psychopathology rather than temperament.

We retain, however, the concept of temperament as earlyemerging biologically-based individual differences in how children and adults select, process, and respond to salient stimuli within their environments $[7,8]$. The idealized definition points to a stable profile with a presumed (also stable) physiological foundation that creates an enduring pattern of thoughts and behaviors that are early appearing and consistent across time and place [9, 10]. In this sense, temperament acts as the bridge between biology and personality [8].

Much of our work has focused on BI, a specific temperament trait first identified and described by Jerome Kagan [11, 12]. BI is evident in early childhood and is marked by an early-emerging and persistent tendency to display fear to novelty $[12,13]$, social reticence with unfamiliar people [11, $13,14]$, and sensitivity to approach-avoidance cues [10]. Although most children are initially wary of novelty, approximately 15-20\% show patterns of reactivity and response extreme enough to meet criteria for BI [7]. BI is first identified, and perhaps is evident in its most "pure" form, in the second and third years of life. This is due to the fact that the child has the behavioral repertoire necessary to express a full range of responses in the face of novel sensory stimuli, uncertain events, and unfamiliar social situations [7], while not quite yet possessing the regulatory processes needed to mask and modulate initial reactivity [15].

$\mathrm{BI}$, in turn, is often preceded by individual differences in reactivity that are evident in the first months of life [16]. When presented with benign, but novel, stimuli such as mobiles, puppets, and audio clips, negatively reactive infants at age 4 months respond with negative vocalizations, vigorous limb movements, and arching of the back. In contrast, infants displaying positive reactivity respond to the same stimuli with smiles, positive vocalizations, and limb movements that are active but not tense. Longitudinal work demonstrates that negative reactivity is linked to the later presence of BI [16, 17], while positive reactivity may be a precursor to temperamental exuberance [18].

Children who displayed BI as toddlers often show poorly regulated social behavior and social reticence in elementary school [14, 19]. This, in turn, increases the likelihood of peer rejection, low self-esteem, and poor social competence [20, 21]. It is this pattern of social development that may set the 
foundation for later social withdrawal and social anxiety [1], as will be discussed below. Long-term studies have noted moderate stability of BI from toddlerhood through middle childhood, adolescence, and early adulthood, with correlations across testing sessions ranging from $r=0.24$ and $r=$ 0.64 [22-24].

The wide range in the stability of BI is in part a consequence of the specific methods used and the population examined [25]. As expected, studies examining short gaps (e.g., one year) show greater stability than studies spanning longer periods of time (e.g., 7+ years) [16]. In addition, studies relying on parental report of BI often show more stability than studies based on laboratory observation of behavior [2]. This may reflect the fact that parents have access to a broader array of opportunities to observe temperamental variation, or alternatively, may echo stable parental conceptualizations of the child [7]. Studies relying on behavioral observation must work to capture stable underlying processes that change in expression across different developmental periods, reflecting heterotypic continuity $[25,26]$.

Of direct interest to the current review, studies focused on individuals with extreme levels of BI show higher levels of stability relative to studies examining levels of BI across broader community samples [2]. It is presumed that children with extreme, stable BI are at the greatest risk for anxiety [1]. Most studies examining the stability of BI or fearful temperament assume linear relations that reflect differential, or rankorder, stability. This assumption may break down at the extremes. Incorporating biological measures systematically across longitudinal studies may help distinguish diverging trajectories in the function and expression of temperament, in terms of both heterotypic continuity and differential stability.

$\mathrm{BI}$ is conceptually similar to a number of constructs often found in the literature, including temperamental shyness, temperamental fearfulness, social reticence, anxious solitude, and social withdrawal $[26,27]$. Although most of the literature does not strictly adhere to these constraints, Kagan's initial characterization of $\mathrm{BI}$ contends that this temperamental category is distinguished by the fact that it is (1) a qualitatively distinct category of temperament that is not simply an end point of a continuum, (2) defined by observed patterns of behavior across contexts and tasks, independent of questionnaire-based reports, and (3) associated with a distinct neurobiological profile rooted in the animal literature [7].

This third point is particularly salient for our current discussion of the association between BI and social anxiety. Kagan drew on a line of neuroscience research noting that the amygdala is functional shortly after birth [28], and is linked to the acquisition of conditioned fear [29], the induction of vigorous limb movements [30], and the modulation of distress cries [31]. Thus, he postulated that the unique pattern of behavior seen in negative reactive infants, which is then associated with $\mathrm{BI}$ in toddlerhood, was a direct reflection of a hyper-responsive amygdalar response to novel and salient environmental stimuli [7]. This amygdalar response, in turn, is then presumed to fuel much of the distinct psychophysiological and behavioral profile noted in BI.

This profile, as discussed below, may also serve as the foundation for the association between early BI and the later emergence of social anxiety disorder [32•]. Indeed, recent work suggests that the amygdala may be central to the structural and functional reorganization of brain networks subserving social processes across development, building on its hub-like architecture with cortical and subcortical neural regions [33]. The clinical literature reinforces this characterization of the amygdala as neuroimaging studies implicate perturbations in a distributed network encompassing the amygdala, the bed nucleus of the stria terminalas (BNST), the striatum, and multiple regions of the prefrontal cortex in pediatric anxiety [34].

\section{Empirical Links between Behavioral Inhibition and Social Anxiety}

BI is associated with increased risk for social anxiety. In prospective research, BI emerges as the strongest individualdifference predictor of risk for later anxiety [35, 36], particularly for children who show consistent signs of BI across multiple testing points $[37,38]$. Across multiple studies and samples, the relation is strongest when specifically examining social anxiety, as opposed to specific phobias and generalized anxiety disorder (GAD) [2]. Kagan and colleagues [39] have argued that children at the extreme may show a unique developmental trajectory as the association between temperament and socioemotional outcomes is nonlinear.

Indeed, Hayward and colleagues [35] found that adolescents rated in the top $15 \%$ of self-reported BI had a fivefold increase in developing social anxiety, relative to peers without an extreme temperamental profile. Kagan and Snidman [9] found that at age 13, $61 \%$ of adolescents identified as BI at age 2 showed signs of social anxiety during interactions with an unfamiliar adult. This is compared with only $27 \%$ of the children in a non-inhibited group. In another longitudinal study, Prior and colleagues [38] found similar associations. Schwartz and colleagues [36] found that adolescents characterized as BI at the age of 2 were more likely than their peers without BI to show symptoms of social anxiety, based on a semi-structured diagnostic interview. Indeed, $61 \%$ of the adolescents with BI had current symptoms, and $80 \%$ had shown anxiety symptoms at one point in their lifetime. We have found a fourfold increase in anxiety by mid-adolescence in children with high, stable levels of BI from age 14 months through age 7 years, evident in diagnostic interviews as well as parent- and self-report measures of anxiety symptoms [4]. These results were supported by a recent meta-analysis by 
Clauss and Blackford [3] indicating that children high in BI show an adjusted odds ratio of 7.59, or a relative risk of 4.12, for developing social anxiety compared to peers without BI.

$\mathrm{BI}$ and social anxiety share a similar psychophysiological, neural, and cognitive profile. The initial studies of BI were motivated by observations of a distinct pattern of behavior manifested by a subset of children when confronted with novelty [7]. The interest in anxiety emerged over time as the data revealed clear links between early BI and social anxiety $[1,3]$. As such, subsequent studies looked to see if children and adolescents with a history of BI also exhibit markers of risk or impairment previously found in clinically anxious adolescents [5•], even in the absence of psychopathology. This last point is particularly important as it contributes to current debate concerning the role of BI as either an endophenotypic marker of risk, a pre-existing diathesis for anxiety, or the prodromal form of anxiety [40].

A systematic series of studies has documented a pattern of functioning in individuals with early BI that parallels earlier work with clinically anxious individuals [41]. For example, children and adolescents with a history of BI show elevated attention to novelty [42, 43] and to behavioral errors [44], and have difficulty disengaging from threat cues [45], as marked by event-related potentials (ERPs). They also exhibit increased potentiated startle to threat [46], greater right frontal electroencephalogram (EEG) asymmetry at rest [47, 48], perturbations in salivary cortisol levels at rest and after provocation $[49,50]$, higher heart rates and lower heart rate variability at rest [51], unique patterns of cardiac reactivity to emotionally-laden narratives [52], and lower thresholds for detecting threat in the environment $[53,54]$.

In each case, the specific functional marker was chosen for study because it was hypothesized to grow out of the hypersensitive limbic response to novelty and uncertainty presumed to fuel the observed behavioral profile of BI [7, 32•]. Although 20 years of work pointed to the role the amygdala may play in $\mathrm{BI}$, direct examination of this brain structure has only recently become available with the widespread adoption of magnetic resonance imaging (MRI) and functional MRI (fMRI) technology for research $[55,56]$.

The first major study of amygdala function in BI found that young adults categorized as inhibited in the second year of life showed significant bilateral amygdalar activation to the presentation of novel faces, versus fixation, relative to participants without a history of BI [57]. We [58] found that adolescents with a history or childhood BI also showed increased amygdala activation when attending to their subjective fear of emotion faces during an attention-emotion face task, coupled with amygdala deactivation when passively viewing the same faces. Intriguingly, McClure and colleagues [59] found the same pattern of hyper-activation and deactivation across conditions in clinically anxious adolescents completing the identical fMRI task.
Given the initial biologically-based formulation of BI drawn from the animal literature, early studies naturally focused on examining amygdala-based patterns of functioning. However, a recent series of studies suggest that BI may be associated with a distributed neural network sensitive to salient environmental cues that also includes regions traditionally associated with reward processing, such as the striatum [40]. Intriguingly, similar reward-linked patterns are also evident in adolescents with current social anxiety disorder [56].

Guyer and colleagues [60] were among the first to show that adolescents with a history of BI showed increased striatal response in anticipation of increasing monetary rewards. Building on this initial finding, we have found that the striatal hyper-response to monetary reward is evident only when reward is contingent on one's own performance [61] and when anticipated feedback is met with a negative response [62]. This heightened striatal response in BI is also linked to increased anxiety, particularly in the context of genetic risk [63], and increased levels of substance use [64]. Adolescents with a history of BI also show striatal hypersensitivity to anticipated social evaluation [65].

It may be that striatal dysfunction reflects worry in the anticipation of uncertain outcomes or concern over performance when the stakes increase [56]. Social anxiety is associated with dysfunctions in the striatal dopaminergic system and altered striatal function. This may constitute an additional shared pathway between BI and social anxiety. Indeed, we have shown heightened striatal response to anticipated reward in adolescents with social anxiety but not GAD [66]. It may be that striatal function is a biomarker that differentiates between these two anxiety diagnoses while the amygdala is involved in anxiety in a non-specific fashion that does not differentiate between the anxiety disorders.

Recent work on the BI-anxiety link has also focused on core cognitive processes as mounting evidence suggests that clear perturbations in information processing may play a central role in the etiology and maintenance of anxiety [67]. This encompasses which aspects of the environment the child attends to, how he or she interprets events in the environment, and how events are consolidated into later memories [68, 69]. Much of the focus has been on attention mechanisms following recent meta-analyses suggesting that attention biases to threat are evident across diagnostic boundaries in anxious children and adults [70] and that actively manipulating attention may lead to a decrease in anxiety symptoms [71]. Of specific interest to the current discussion, socially anxious adolescents show attention bias to threat relative to nonanxious peers [72] and display a distinct pattern of limbic and frontal activation during an fMRI task assessing attention bias to threat $[73,74]$. In addition, early patterns of attention are associated with increasing levels of BI and social withdrawal over the course of childhood and adolescence [75]. 
The initial study of attention bias in BI found that adolescents with a childhood history of BI, in the absence of an anxiety diagnosis, also showed increased attention bias to threat relative to their non-BI peers [76]. In addition, early $\mathrm{BI}$ was associated with elevated levels of social withdrawal in adolescence only if the individual also showed attention bias to threat. We found the same pattern of moderation in an independent sample of high BI children at age 5 [77]. As young adults, individuals characterized in childhood with BI exhibited greater negative functional connectivity between the amygdala and two frontal regions, the dorsolateral prefrontal cortex and anterior insula [78], during the same fMRI attention-bias task completed by clinically anxious adolescents [73]. Increased connectivity strength, in turn, was associated with increased levels of internalizing symptoms among the participants with a history of BI [78].

\section{Theoretical Links between Behavioral Inhibition and Social Anxiety}

Questions have been raised regarding the nature of the relation between $\mathrm{BI}$ and social anxiety. Although there is strong agreement that BI and social anxiety are consistently linked at multiple levels of analysis, there remains discussion concerning the potential causal relations between the two constructs. Some researchers have taken a definitive stand on the issue. For example, Fox and colleagues have stated that $\mathrm{BI}$ is a temperament "identified early in infancy and not an early manifestation of disorder" (p. 789) [79]. However, others have argued that the relation between $\mathrm{BI}$ and anxiety is more fluid, particularly if they are approaching the question from the perspective of pediatric psychiatry [80]. Klein [6 $\left.6^{\bullet}\right]$ notes that there are broad categories of models in the literature (Fig. 1).

The first model suggests that BI and social anxiety are linked by a shared common causal influence; BI does not cause anxiety. The second model emphasizes a functional continuity between BI and anxiety, arguing that they are simply different points within a single spectrum. One formulation of this continuity model implies that individuals display a fixed clinical expression, remaining fairly static in one region of the spectrum. Another form of the continuity model proposes that $\mathrm{BI}$ is part of a single developmental sequence in which the trajectory progresses into disorder as the child "travels" across the spectrum. From this perspective, BI is a subthreshold form of social anxiety, a prodromic marker for the acute disorder.

In contrast, the third set of models suggests that while BI is a separate construct from social anxiety, it can predispose an individual to anxiety or influence the form and course of the disorder. In one formulation, $\mathrm{BI}$ is characterized as a diathesis that may give rise to disorder in the presence of a second moderating risk factor [40]. For example, BI will manifest an association with anxiety when coupled with over-solicitous parenting [81]. Alternatively, BI may shape how an initial risk factor evolves into disorder. For example, children high in negative affect may show increased social anxiety if also high in BI, but would otherwise be at risk for increased depression in the absence of BI.

There is little to no systematic study directly examining these models. Indeed, for the most part they are implied within the discussion of a specific study or analysis, rather than addressed as an explicit formulation of the question at hand. Distinguishing between models is also difficult in light of current debate regarding the best way to define, categorize, and identify discrete psychological disorders.

One striking distinction between BI and social anxiety stems from core differences in how the constructs are conceptualized and defined. Based on Kagan's initial work [11, 12], $\mathrm{BI}$ is rooted in observed patterns of behavior in response to novelty that are directly associated with heightened levels of limbic reactivity [7]. In contrast, social anxiety as defined by DSM-5 is identified via reported patterns of avoidance and distress in situations involving social performance and is agnostic with respect to underlying etiology [67]. Kagan [7] took pains to highlight the distinction between $\mathrm{BI}$ and anxiety in his statement that "BI does not refer to displays of distress when exposed to pain or dangerous events, only to timidity in unexpected or unfamiliar experiences" (p. 69-70).

Differences in emotion regulation may distinguish between BI children with and without social anxiety. In addition to Kagan's model of BI, the other most influential temperament model has been articulated by Mary Rothbart [8]. Her model postulates two main components of temperament-initial emotional, psychophysiological, and behavioral responses to the environment, reactivity, and the subsequent ability to modulate initial tendencies by heightening or inhibiting responses, regulation. As noted above, children who go on to show high levels of BI tend to show negative reactivity to novelty as infants [7]. A review of the literature examining the link between $\mathrm{BI}$ and anxiety suggests that much of the focus to date has been on the reactive components of temperament [32].

These studies have found marked similarities across multiple levels of analysis and help explain the fourfold increase in social anxiety seen in children with BI versus peers without BI [3]. However, we also know that the majority of children with BI do not go on to experience levels of social anxiety that rise to the level of disorder [25, 82]. Thus, there are likely moderating factors that shape the developmental trajectory between early BI and later anxiety [68]. It may be that children with a history of BI who can marshal effective regulatory processes in the face of heightened reactivity constitute the population of temperamentally at-risk but healthy children and adults [83]. 
For example, Lonigan and Vasey [84] found that attention biases to threat did not lead to elevated levels of anxiety if children also exhibited high levels of effortful control. Effortful control, in turn, can be defined as the self-regulatory component of temperament linked to the ability to modulate reactivity, monitor behavior, and engage in future planning [15]. The relation between reactivity, regulation, and social withdrawal is dissociable as threat bias moderates the significant positive link between reactivity and social withdrawal but does not modulate the direct negative association between effortful control and social withdrawal [85]. Effortful control is seen as a core tool in the child's arsenal needed to both selfregulate and integrate oneself as an adaptive member of the larger social environment.

However, to complicate matters a bit, effortful control is itself comprised by a number of sub-components that may have varying impact on the relation between BI and anxiety. In particular, the data indicate that while attentional control may ameliorate temperamental risk, high levels of inhibitory control, in the context of BI, may actually exacerbate risk for social anxiety [86]. The ability to flexibly deploy attention and navigate away from threat may head off negative affect, buffering subsequent cognitive and behavioral responses. This aligns with recent empirical and theoretical work suggesting that early attention processes shape broad patterns of socioemotional functioning $[68,87 \cdot, 88]$.

In contrast, inhibitory control is the ability to inhibit a dominant response in the service of performing a subdominant response [89]. In children prone to dysregulated responses to the environment, such as in ADHD and conduct disorder, increases in inhibitory control are associated with improved outcomes [90]. However, among children with BI, inhibitory control may lead to an excessive level of behavioral monitoring and may actually facilitate worry [91]. Indeed, multiple studies have found that the presence of elevated inhibitory control increases the likelihood that individuals with early BI will also show elevated levels of anxiety [44, 86, 92].

\section{Outstanding Issues and Future Directions}

There is a clear literature strongly linking early patterns of BI to the later emergence of social anxiety. This association is, in turn, associated with a distinct behavioral and psychobiological profile. However, much of this work is still descriptive in nature and is unable to directly assess the mechanisms that fuel observed relations.

Research attempting to bridge many of the outstanding issues regarding the link between $\mathrm{BI}$ and anxiety has been hampered by a set of structural constraints. First, as Bates [5•] and Klein $\left[6^{\bullet}\right]$ noted, many studies first identify a sample once discrete symptoms of anxiety have already emerged. As a result, it is often hard to extract clear measures of temperament from retrospective report of thoughts, moods, and behavior. In this respect, prospective longitudinal studies are in the best position to capture the developmental trajectory of early temperament and any anxiety-associated sequellae $[5 \bullet, 6 \bullet]$. However, these studies are few and far between.

Indeed, in carrying out their recent meta-analysis examining the empirical link between BI and social anxiety disorder, Clauss and Blackford [3] found only seven studies that met all of their criteria for inclusion. The expense, complexity, and long time-window involved suggest that the number of studies available is unlikely to increase significantly any time soon. Finally, among the available studies the number of participants with diagnosed social anxiety is fairly small, even with the elevated level of risk [4, 42, 44, 46, 54, 93]. Thus, it is difficult, if not impossible, to fully populate the needed cells of participants with and without childhood BI crossed with participants with and without social anxiety disorder. This last problem may be alleviated somewhat as researchers step back from traditional research designs reliant on diagnostic categories and incorporate instead continuous measures of underlying anxiogenic processes, as advocated by the National Institute of Mental Health in the research domain criteria [94].

Work is also needed to distinguish BI from the other causal agents that lead to social anxiety, some of which may co-occur with BI. For example, there are clear data linking parenting styles [67], unstable or negative peer relations [95], environmental stress [25], and heightened rejection sensitivity [96] with pediatric anxiety. Each of these mechanisms may operate independently to generate anxiety. In addition, BI may act to elicit environmental input that exacerbates risk [2]. Research assessing these inter-related causal factors is only now emerging.

At the same time, studies examining the impact of temperament on psychopathology is moving beyond the initial focus on BI (e.g., exuberance leading to externalizing difficulties [18]). Even in the case of social anxiety, BI is not the only temperament trait associated with elevated levels of this disorder. For example, Buss and colleagues $[27,97,98 \bullet, 99]$ have characterized a separate fearful temperament, dysregulated fear (DF), that is defined as "behavioral responses that are not matched to the incentive properties of the eliciting context or the availability of coping resources" (p. 50) [27]. In contrast, BI is often characterized by aggregating behavioral measures across contexts with a focus on highly novel situations. Buss has found that while $\mathrm{BI}$ and $\mathrm{DF}$ are highly correlated $(r=0.60)$, this relation is mainly carried by children low in both BI and DF. In addition, the link between DF and social anxiety is evident above and beyond individual levels of BI [27]. This line of research suggests that the level of instability in BI over time and the heterogeneity in psychiatric outcome may reflect variation in temperament among children initially labeled as BI. 
Kagan [100] has suggested that "contemporary scientists resemble children who can only read six words trying to read a Harry Potter novel" (p.162). Thirty years of research has slowly added to our emerging vocabulary as we attempt to integrate information across multiple levels of analysis that span both place and time. Currently, much of our knowledge skips from the first years of life to adolescence, where we see the first jump in psychopathology. The next 30 years will need to focus on bridging these gaps and extending our understanding into adulthood to see if the imprints of early temperament, shaped by the unique stressors and opportunities of adolescence, continue to impact long-term patterns of functioning across the life course.

Acknowledgment Support for manuscript preparation was provided by grants from the National Institutes of Health (MH\# 094633 and MH\# 103627) and The Pennsylvania State University Social Science Research Institute (Level II Grant) to Koraly Pérez-Edgar and the National Institutes of Health (MH\# 093605 and MH\# 098370) and the William T. Grant Foundation to Amanda E. Guyer.

\section{Compliance with Ethics Guidelines}

Conflict of Interest Amanda Guyer served as a consultant for the William T Grant Foundation, and is employed with the Society for Research on Child Development. Guyer received travel support to present at the annual meeting of the American College of Neuropsychopharmacology. Koraly Perez-Edgar served as a consultant for the NIH and the American Psychological Association. Perez-Edgar has grants pending from the National Institute of Mental Health; received honoraria from the University of Colorado Anschutz Medical Campus, Denver, CO; received travel support from the Jacobs Foundation and the Child Mind Institute.

Human and Animal Rights and Informed Consent This article does not contain any studies with human or animal subjects performed by the authors.

\section{References}

Papers of particular interest, published recently, have been highlighted as:

- Of importance

1. Fox NA, Pine DS. Temperament and the emergence of anxiety disorders. J Am Acad Child Adolesc Psychiatry. 2012;51:125-8.

2. Pérez-Edgar K, Fox NA. Temperament and anxiety disorders. Child Adolesc Psychiatr Clin N Am. 2005;14:681-706.

3. Clauss JA, Blackford JU. Behavioral inhibition and risk for developoing social anxiety disorder: A meta-analytic study. J Am Acad Child Adolesc Psychiatry. 2012;51:1066-75.

4. Chronis-Tuscano A, Degnan KA, Pine DS, et al. Stable behavioral inhibition during infancy and early childhood predicts the development of anxiety disorders in adolescence. J Am Acad Child Adolesc Psychiatry. 2009;48:928-35.

5. Bates JE, Schermerhorn AC, Petersen IT. Temperament Concepts in Developmental Psychopathology. In: Lewis MD, Rudolph KD, editors. Handbook of Developmental Psychopathology. New York: Springer; 2014. The authors examine the role temperament traits may play in the emergence of developmental psychopathology. They also compare the different temperament models proposed in the literature.

6. Klein DN, Dyson MW, Kujawa A, et al. Temperament and internalizing disorders. In: Zentner M, Shiner RL, editors. Handbook of Temperament. New York: Guilford; 2012. This chapter reviews the broader literature examining the association between temperament, in its various forms, with the broad spectrum of psychopathology in children.

7. Kagan J. The biography of behavioral inhibition. In: Zentner M, Shiner RL, editors. Handbook of temperament. New York: Guilford Press; 2012. p. 69-82.

8. Rothbart MK. Advances in temperament: History, concepts, and measures. In: Zentner M, Shiner RL, editors. Handbook of temperament. New York: Guilford Press; 2012. p. 3-20.

9. Kagan J, Snidman N, McManis M, et al. Temperamental contributions to the affect family of anxiety. Psychiatr Clin N Am. 2001;24(4):677-88.

10. Fox NA, Henderson HA, Pérez-Edgar K, et al. The biology of temperament: An integrative approach. In: Nelson C, Luciana M, editors. The Handbook of Developmental Cognitive Neuroscience. Cambridge: MIT Press; 2008. p. 839-54.

11. Garcia Coll C, Kagan J, Reznick JS. Behavioral inhibition in young children. Child Dev. 1984;55:1005-19.

12. Kagan J, Reznick JS, Clarke C, et al. Behavioral inhibition to the unfamiliar. Child Dev. 1984;55(6):2212-25.

13. Fox NA, Henderson HA, Marshall PJ, et al. Behavioral inhibition: linking biology and behavior within a developmental framework. Annu Rev Psychol. 2005;56:235-62.

14. Coplan RJ, Rubin KH, Fox NA, et al. Being alone, playing alone, and acting alone: distinguishing among reticence and passive and active solitude in young children. Child Dev. 1994;65:129-37.

15. Rueda MR. Effortful Control. In: Zentner M, Shiner RL, editors. Handbook of temperament. New York: Guilford Press; 2012. p. $145-68$.

16. Fox NA, Henderson HA, Rubin KH, et al. Continuity and discontinuity of behavioral inhibition and exuberance: Psychophysiological and behavioral influences across the first four years of life. Child Dev. 2001;72:1-21.

17. Kagan J, Snidman N. Infant predictors of inhibited and uninhibited profiles. Psychol Sci. 1991;2:40-4.

18. Degnan KA, Hane AA, Henderson HA, et al. Longitudinal stability of temperamental exuberance and social-emotional outcomes in early childhood. Dev Psychol. 2011;47:765-80.

19. Fox NA, Rubin KH, Calkins SD, et al. Frontal activation asymmetry and social competence at four years of age. Child Dev. 1995;66:1771-84.

20. Rubin K, Chen X, Hymel S. Socioemotional characteristics of withdrawn and aggressive children. Merrill-Palmer Q. 1993;39: 518-34.

21. Schmidt LA, Schulkin J. Extreme fear, shyness, and social phobia: origins, biological mechanisms, and clinical outcomes. New York: Oxford University Press; 1999.

22. Goldsmith HH, Lemery KS. Linking temperamental fearfulness and anxiety symptoms: a behavior-genetic perspective. Biol Psychiatry. 2000;48:1199-209.

23. Degnan KA, Henderson HA, Fox NA, et al. Predicting social wariness in middle childhood: The moderating roles of child care history, maternal personality, and maternal behavior. Soc Dev. 2008; 17:471-87.

24. Blackford JU, Avery SN, Cowan RL, et al. Sustained amygdala response to both novel and newly familiar faces characterizes inhibited temperament. Soc Cogn Affect Neurosci. 2011;6:621-9. 
25. Degnan KA, Fox NA. Behavioral inhibition and anxiety disorders: Multiple levels of a resilience process. Dev Psychopathol. 2007;19:729-46.

26. Fu X, Perez-Edgar K. Theories of Temperament Development. In: Wright JD, editor. The International Encyclopedia of Social \& Behavioral Sciences. Amsterdam: Elsevier; 2014.

27. Buss KA, Kiel EJ. Temperamental risk factors for pediatric anxiety disorders. In: Vasa RA, Roy AK, editors. Pediatric Anxiety Disorders: A Clinical Guide. New York: Springer; 2013. p. 47-68.

28. Benes FM. Development of the corticolimbic system. In: Dawson G, Fischer K, editors. Human Behavior and the Developing Brain. New York: Guilford Press; 1994.

29. Davis M, Walker DL, Lee Y. Amygdala and bed nucleus of the stria terminalis: differential roles in fear and anxiety measured with the acoustic startle reflex. Phil Trans R Soc London Series B Biol Sci. 1997;352:1675-87.

30. Amaral D. The amygdala, social behavior, and danger detection. Ann N Y Acad Sci. 2003;1000:337-47.

31. Kagan J, Snidman N. The long shadow of temperament. Cambridge: Belknap Press of Harvard University Press; 2004.

32. White LK, Lamm C, Helfinstein SM, et al. Neurobiology and neurochemistry of temperament in children. In: Zentner M, Shiner RL, editors. Handbook of Temperament. New York: Guilford; 2012. This chapter reviews the biological bases of temperament, incorporating data from the animal literature, developmental psychology, and neuroscience. The text highlights the myriad levels of analysis implicated in observed patterns of behavior.

33. Scherf KS, Smyth JM, Delgado MR. The amygdala: An agent of change in adolescent neural networks. Horm Behav. 2013;64: 298-313.

34. Blackford JU, Pine DS. Neural substrates of childhood anxiety disorders: A review of neuroimaging findings. Child Adolesc Psychiatr Clin N Am. 2012;21:501-25.

35. Hayward C, Killen JD, Kraemer HC, et al. Linking self-reported childhood behavioral inhibition to adolescent social phobia. J Am Acad Child Adolesc Psychiatry. 1998;37:1308-16.

36. Schwartz CE, Snidman N, Kagan J. Adolescent social anxiety as an outcome of inhibited temperament in childhood. J Am Acad Child Adolesc Psychiatry. 1999;38:1008-15.

37. Biederman J, Rosenbaum JF, Bolduc-Murphy EA, et al. A 3-year follow-up of children with and without behavioral inhibition. J Am Acad Child Adolesc Psychiatry. 1993;32:814-21.

38. Prior M, Smart D, Sanson A, et al. Does shy-inhibited temperament in childhood lead to anxiety problems in adolescence? J Am Acad Child Adolesc Psychiatry. 2000;39(4):461-8.

39. Kagan J, Snidman N, McManis M, et al. One measure, one meaning: Multiple measures, clearer meaning. Dev Psychopathol. 2002;14(3):463-75.

40. Caouette JD, Guyer AE. Gaining insight into adolescent vulnerability for social anxiety from developmental cognitive neuroscience. Dev Cogn Neurosci. 2014;8:65-76.

41. Fox NA, Hane AA, Pérez-Edgar K. Psychophysiological methods for the study of developmental psychopathology. In: Cicchetti D, Cohen DJ, editors. Developmental psychopathology. Hoboken: Wiley; 2006. p. 381-426.

42. Reeb-Sutherland BC, Vanderwert RE, Degnan KA, et al. Attention to novelty in behaviorally inhibited adolescents moderates risk for anxiety. J Child Psychol Psychiatry. 2009;50:136572.

43. Marshall PJ, Reeb BC, Fox NA. Electrophysiological responses to auditory novelty in temperamentally different 9-month-old infants. Dev Sci. 2009;12:568-82.

44. McDermott JM, Pérez-Edgar K, Henderson HA, et al. A history of childhood behavioral inhibition and enhanced response monitoring in adolescence are linked to clinical anxiety. Biol Psychiatry. 2009;65:445-8.
45. Pérez-Edgar K, Fox NA. A behavioral and electrophysiological study of children's selective attention under neutral and affective conditions. J Cogn Dev. 2005;6(1):89-118.

46. Reeb-Sutherland BC, Helfinstein SM, Degnan KA, et al. Startle modulation in behaviorally inhibited adolescents with a lifetime occurrence of anxiety disorders. J Am Acad Child Adolesc Psychiatry. 2009;48:610-7.

47. Calkins SD, Fox NA, Marshall TR. Behavioral and physiological antecedents of inhibited and uninhibited behavior. Child Dev. 1996;67:523-40.

48. Hane AA, Fox NA, Henderson HA, et al. Behavioral reactivity and approach-withdrawal bias in infancy. Dev Psychol. 2008;44: 1491-6.

49. Schmidt LA, Fox NA, Sternberg EM, et al. Adrenocortical reactivity and social competence in seven year-olds. Personal Individ Differ. 1999;26(6):977-85.

50. Pérez-Edgar K, Schmidt LA, Henderson HA, et al. Salivary cortisol levels and infant temperament shape developmental trajectories in boys at risk for behavioral maladjustment. Psychoneuroendocrinology. 2008;33:916-25.

51. Marshall PJ, Stevenson-Hinde J. Behavioral inhibition, heart period, and respiratory sinus arrhythmia in young children. Dev Psychobiol. 1998;33(3):283-92.

52. Bar-Haim Y, Fox NA, VanMeenen KM, et al. Children's narratives and patterns of cardiac reactivity. Dev Psychobiol. 2004;44:238-49.

53. LoBue V, Pérez-Edgar K. Sensitivity to social and non-social threats in temperamentally shy children at-risk for anxiety. Dev Sci. 2014;17:239-47.

54. Reeb-Sutherland BC, Williams LR, Degnan KA, et al. Identification of emotional facial expressions among behaviorally inhibited adolescents with lifetime anxiety disorders. Cognition \& Emotion, in press.

55. Schwartz CE, Rauch SL. Temperament and its implications for neuroimaging of anxiety disorders. CNS Spectrums. 2004;9:284 91.

56. Guyer AE, Masten CL, Pine DS. Neurobiology of pediatric anxiety disorders. In: Vasa RA, Roy AK, editors. Pediatric Anxiety Disorders: A Clinical Guide. New York: Springer; 2013.

57. Schwartz CE, Wright CI, Shin LM, et al. Inhibited and uninhibited infants "grown up": Adult amygdalar response to novelty. Science. 2003;300:1952-3.

58. Pérez-Edgar K, Roberson-Nay R, Hardin MG, et al. Attention alters neural responses to evocative faces in behaviorally inhibited adolescents. NeuroImage. 2007;35:1538-46.

59. McClure EB, Monk CS, Nelson EE, et al. Abnormal attention modulation of fear circuit function in pediatric generalized anxiety disorder. Arch Gen Psychiatry. 2007;64:109-16.

60. Guyer AE, Nelson EE, Perez-Edgar K, et al. Striatal functional alteration in adolescents characterized by early childhood behavioral inhibition. J Neurosci. 2006;26(24):6399-405.

61. Bar-Haim Y, Fox NA, Benson B, et al. Neural correlates of reward processing in adolescents with a history inhibited temperament. Psychol Sci. 2009;20:1009-18.

62. Helfinstein SM, Benson B, Pérez-Edgar K, et al. Striatal responses to negative monetary outcomes differ between behaviorally inhibited and non-inhibited adolescents. Neuropsychologia. 2011;49:479-85.

63. Pérez-Edgar K, Hardee J, Guyer AE, et al. DRD4 and striatal modulation of the link between childhood behavioral inhibition and adolescent anxiety. Soc Cogn Affect Neurosci. 2014;9:44553.

64. Lahat A, Pérez-Edgar K, Degnan KA, et al. Early childhood temperament predicts substance use in young adults. Transl Psychiatry. 2012;2:e157.

65. Guyer AE, Benson B, Choate VR, et al. Lasting associations between early-childhood temperament and late-adolescent 
reward-circuitry response to peer feedback. Dev Psychopathol. 2014;26:229-43.

66. Guyer AE, Choate VR, Detloff A, et al. Striatal functional alteration during incentive anticipation in pediatric anxiety disorders. Am J Psychiatr. 2012;169:205-12.

67. Vasey M, Bosmans G, Ollendick TH. The developmental psychopathology of anxiety. In: Lewis MD, Rudolph KD, editors. Handbook of Developmental Psychopathology. New York: Springer; 2014

68. Pérez-Edgar K, Taber-Thomas B, Auday E, et al. Temperament and attention as core mechanisms in the early emergence of anxiety. In: Lagattuta K, editor. Children and Emotion: New Insights into Developmental Affective Science. Freiburg: Karger; 2014. p. 42-56.

69. White LK, Helfinstein SM, Fox NA. emperamental Factors Associated with the Acquisition of Information Processing Biases and Anxiety. In: Hadwin JA, Field AP, editors. Information Processing Biases and Anxiety: A Developmental Perspective. Chichester: Wiley; 2010. p. 233-52.

70. Bar-Haim Y, Lamy D, Pergamin L, et al. Threat-related attentional bias in anxious and nonanxious individuals: A meta-analytic study. Psychol Bull. 2007;133:1-24.

71. Hakamata Y, Lissek S, Bar-Haim Y, et al. Attention Bias Modification Treatment: A meta-analysis towards the establishment of novel treatment for anxiety. Biol Psychiatry. 2010;68: 982-90.

72. Roy AK, Vasa RA, Bruck M, et al. Attention bias toward threat in pediatric anxiety disorders. J Am Acad Child Adolesc Psychiatry. 2008;47:1189-96.

73. Monk CS, Nelson EE, McClure EB, et al. Ventrolateral prefrontal cortex activation and attention bias in responsive to angry faces in adolescents with generalized anxiety disorder. Am J Psychiatr. 2006;163:1091-7.

74. Monk CS, Telzer EH, Mogg K, et al. Amygdala and ventrolateral prefrontal cortex activation to masked angry faces in children and adolescents with generalized anxiety disorder. Arch Gen Psychiatry. 2008;65:568-76.

75. Pérez-Edgar K, McDermott JM, Korelitz K, et al. Patterns of sustained attention in infancy shape the developmental trajectory of social behavior from toddlerhood through adolescence. Dev Psychol. 2010;46:1723-30.

76. Pérez-Edgar K, Bar-Haim Y, McDermott JM, et al. Attention biases to threat and behavioral inhibition in early childhood shape adolescent social withdrawal. Emotion. 2010;10:34957.

77. Pérez-Edgar K, Reeb-Sutherland BC, McDermott JM, et al. Attention biases to threat link behavioral inhibition to social withdrawal over time in very young children. J Abnorm Child Psychol. 2011;39:885-95.

78. Hardee J, Benson B, Bar-Haim Y, et al. Patterns of neural connectivity during an attention bias task moderate associations between early childhood temperament and internalizing symptoms in young adulthood. Biol Psychiatry. 2013;74:273-9.

79. Fox NA, Barker TV, White LK, et al. Commentary: To intervene or not? Appreciating or treating individual differences in childhood temperament-remarks on Rapee (2013). J Child Psychol Psychiatry. 2013;54:789-90.

80. Beesdo K, Knappe S, Pine DS. Anxiety and anxiety disorders in children and adolescents: Developmental issues and implications for DSM-V. Psychiatr Clin N Am. 2009;32: 483-524.

81. Williams LR, Degnan KA, Pérez-Edgar K, et al. Impact of behavioral inhibition and parenting style on internalizing and externalizing problems from early childhood through adolescence. J Abnorm Child Psychol. 2009;37:1063-75.
82. Degnan KA, Almas AN, Fox NA. Temperament and the environment in the etiology of childhood anxiety. J Child Psychol Psychiatry. 2010;51:497-517.

83. Jarcho JM, Fox NA, Pine DS, et al. The neural correlates of emotion-based cognitive control in adults with early childhood behavioral inhibition. Biol Psychol. 2013;92:306-14.

84. Lonigan C, Vasey M. Negative affectivity, effortful control, and attention to threat-relevant stimuli. J Abnorm Child Psychol. 2009;37:387-99.

85. Cole C. Attention Biases to Threat Link Components of Temperament to Social Withdrawal in Very Young Children. Fairfax: George Mason University; 2011.

86. White LK, McDermott JM, Degnan KA, et al. Behavioral inhibition and anxiety: The moderating roles of inhibitory control and attention shifting. J Abnorm Child Psychol. 2011;39:735-47.

87. LoBue V. What are we so afraid of? How early attention shapes our most common fears. Child Dev Perspect. 2013;7:38-42. This article highlights an exciting new area of research suggesting that early attention mechanisms may shape broad patterns of socioemotional behavior.

88. Todd RM, Cunningham WA, Anderson AK, et al. Affect-biased attention as emotion regulation. Trends Cogn Sci. 2012;16:36572.

89. Rothbart MK, Rueda MR. The development of effortful control. In: Posner I, Mayr U, Awh E, Keele S, editors. Developing individuality in the human brain: A tribute to Michael. Washington, D.C: American Psychological Association; 2005. p. 167-88.

90. Beauchaine TP, Gatzke-Kopp L. Instantiating the multiple levels of analysis perspective in a program of study on externalizing behavior. Dev Psychopathol. 2012;24:1003-18.

91. Henderson HA. Electrophysiological correlates of cognitive control and the regulation of shyness in children. Dev Neuropsychol. 2010;35:177-93.

92. Thorell LB, Bohlin G, Rydell A-M. Two types of inhibitory control: Predictive relations to social functioning. Int $\mathrm{J}$ Behav Dev. 2004;28:193-203.

93. Shechner T, Britton JC, Pérez-Edgar K, et al. Attention biases, anxiety, and development: Toward or away from threats or rewards? Depression Anxiety. 2012;29:282-94.

94. Sanislow CA, Pine DS, Quinn KJ, et al. Developing constructs for psychopathology research: Research domain criteria. J Abnorm Psychol. 2010;119:631-9.

95. Rubin KH, Hymel S, Mills RSL, et al. Conceptualizing different developmental pathways to and from social isolation in childhood. In: Cicchetti D, Toth S, editors. Internalizing and externalizing expressions of dysfunction. New York, NY: Psychology Press; 2014. p. 91-122.

96. Downey G, Lebolt A, Rincon C, et al. Rejection sensitivity and children's interpersonal difficulties. Child Dev. 1998;69:1074-91.

97. Buss KA. Which fearful toddlers should we worry about? Context, fear regulation, and anxiety risk. Dev Psychol. 2011;47:804-19.

98. Buss KA, Davis EL, Kiel EJ, et al. Dysregulated fear predicts social wariness and social anxiety symptoms during kindergarten. J Child Clin Adolesc Psychol. 2013;42:603-16. This article highlights the complex relation between temperament and social anxiety, highlighting a trait that is both associated with and distinct from BI.

99. Kiel EJ, Buss KA. Prospective relations among fearful temperament, protective parenting, and social withdrawal: The role of maternal accuracy in a moderated mediation framework. J Abnorm Child Psychol. 2011;39:953-66.

100. Kagan J. Behavioral inhibition as a risk factor for psychopathology. In: Beauchaine TP, Hinshaw SP, editors. Child and adolescent psychopathology. Hoboken: Wiley; 2008. p. 157-79. 